\title{
THE AETIOLOGY OF IDIOGLOSSIA
}

\author{
BY \\ I. J. WOLF, M.D., \\ Associate Paediatrician, Barnert Hospital, Paterson, N.J.
}

The term idioglossia was introduced by Hale White and Golding Bird ${ }^{1}$ in 1891 to describe a defective condition of speech in two brothers aged tenand-a-half and nine years respectively, which gave the impression of an individual language. Idioglossia may be defined as lalling or dyslalic speech which is so extreme in degree as to render it incomprehensible to the ordinary listener. A parent or one accustomed to hearing it spoken by a particular child may learn to comprehend it; but the idioglossic speech of another child will not necessarily be understood without study. Various authors have attributed this speech manifestation to:-

1. a faulty perception of speech, the child merely repeating the sounds as he hears them $\left(\right.$ McReady $\left.^{2}\right)$;

2. a faulty motor co-ordination of articulation due to a brain defect of the motor speech centre $\left(\right.$ Hadden $^{3}$, Thomas $\left.{ }^{4}\right)$;

3. a faulty motor co-ordination of articulation secondary to defective intelligence (Wyllie ${ }^{5}$ ); and

4. a faulty auditory perception due to faulty development of the auditory speech centre (Kerr' ${ }^{6}$, Guthrie ${ }^{7}$, Worster-Drought and Allen ${ }^{8}$ ).

These various theories suggest that idioglossia has a varied aetiology, which will be seen to be the case on careful analysis. Recently, however, Worster-Drought and Allen have attempted to establish the thesis that idioglossia is peculiar to congenital auditory imperception (congenital worddeafness) and that those cases reported in the literature in the past fifty years which have been ascribed to other causes have been erroneously interpreted. They have attempted to discredit such aetiologic factors as motor articulatory inco-ordination and mental deficiency, assuming that the latter, for instance, was the result of deprivation rather than the exciting cause.

This stand has influenced Morison ${ }^{9}$ and the Barton-Halls ${ }^{10}$ to believe that idioglossia is characteristic of congenital auditory imperception alone. Further, it has led these writers into reporting certain cases as congenital auditory imperception which do not belong in this category. For instance, the Barton-Halls reported three cases of idioglossia as cases of congenital auditory imperception, one of which certainly was a case of motor dyslalia and another possibly due to mild mental retardation.

Idioglossia is manifest in the speech of children other than those afflicted with congenital auditory imperception. It is the purpose of this paper to outline the varied aetiology of idioglossia and to show that it is merely a stage 
in the development of speech whether this be normal or delayed. The stages of speech development in the normal child may be outlined here.

The first step is crying which is initiated reflexly at birth. Changes in temperature, sudden shifts in position, pain and hunger cause the infant to express his general feeling tone by means of different cries. By the second month usually, in moments of comfort, the infant discovers spontaneously many primitive sounds such as gurgling, cooing and crowing, which are comparable to the reflex cries described to express his unpleasant states of feeling tone. They are produced by accidental, inco-ordinated movements of the muscles of articulation as a result of simple motor discharges. A persistence of the motor patterns laid down associated with pleasurable moments cause the infant to indulge in repetitions.

About the age of eight to ten months, the infant begins to perceive simple and then more complex sounds which he tries to imitate in a meaningless fashion known as echolalia. The associations made between sounds perceived and objects seen, felt and smelled is the basis for the comprehension of speech. The first associations are primitive relating to affective states of hunger, pain and pleasure, and the timbre of voice. Then the infant learns to appreciate the meaning of the combination of sounds comprising words. When an infant responds correctly to an acoustic impression, one may say that he has acquired the understanding of the spoken word. Coincident with the comprehension of speech is the appreciation of facial expressions and gestures.

The comprehension of speech is acquired long before the acquisition of articulate speech, although the infant uses the cry and other sounds to express himself when he has not words at his command. The time comes when he consciously associates a word uttered with its object. If he calls his mother, he associates the word with her and does not repeat it without meaning. This is used as the criterion for the onset of speech; it is usually observed at about the age of one-and-a-half years, although many children reach this stage sooner.

At this point the idioglossic stage of speech development begins. The child tries to reproduce the speech he hears, but a lack of skill in articulation causes him to be dyslalic. In the beginning this is so marked that his utterances are incomprehensible, except for words that are repeated singly. This stage may be termed physiological idioglossia. Slowly his articulation becomes more clear and he passes into a stage of universal dyslalia which is characterized by omissions and reductions, reduplications, assimilations, metatheses, etc. Similar phenomena are observed in the development of languages and dialects as the result of various influences to bring about phonetic change. The same causes are operative to produce these phenomena in the speech of the child as in all spoken language. The fact that the child's articulatory experience is limited makes his speech more labile to these influences. As his skill in articulation increases, the number of faulty articulations decrease. By the third year he is able to express himself clearly in a simple manner. One or two single sound defects may persist for a longer time constituting a partial dyslalia. This term is used in contradistinction to universal dyslalia which denotes a more universally defective speech.

The child in his normal acquisition of speech is seen to pass through the following stages of development:-

1. Crying. 2. Babbling, or the articulation of primitive sounds. 3. Echolalia, or the meaningless repetition of sounds. 4. Comprehension of speech. 5. Aicquisition of speech: a. idioglossia, b. universal dyslalia, and c. partial dyslalia.

Retarded speech development. - For the normal development of speech an intact speech arc must exist. The organs of sight and hearing, the auditory and motor memory centres, the cerebral cortex and the various pathways connecting these areas and organs must be intact. Interruption 
of the arc at any point interferes with the development of speech. If the infant is congenitally deaf, or hearing is lost before speech is well established, deaf mutism results. If hearing becomes impaired due to otitis media or meningitis, speech is improperly received and faulty reproduction ensues. If mental deficiency exists, the child has not the cortical capacity to acquire speech, or speech is delayed in onset and development. If the auditory or motor memory centres are congenitally affected, auditory or kinesthetic speech memories cannot be stored properly. Psychological factors may exist to prolong the development of speech. The causes of retarded speech development may be given as follows:--

1. Psychological factors. 2. Impaired hearing. 3. Mental retardation. 4. Congenital auditory imperception (congenital word-deafness). 5. Congenital articulatory dyskinesthesia (motor dyslalia).

The speech manifestations of retarded lingual development are the same as those of normal lingual development. The stages of development of the lingually retarded child are also comparable to those of the child whose lingual development is normal. The distinction lies in the time of onset of speech and the time required to accomplish normal speech. Depending on the cause, normal speech may never be acquired. In the extreme degree, speech may not advance beyond the stage of primitive sounds or animal-like noises. In less severe cases, speech may remain idioglossic, dyslalic or partially dyslalic. The following examples illustrate the various stages of retarded lingual development that may be observed in mentally deficient children. Comparable stages of development may be observed in any case of retarded lingual development irrespective of the cause.

A child who showed severe mental retardation and was classed as an idiot only understood a few simple commands and expressed his wants and dislikes by means of primitive cries and sounds. Another whose mental retardation was less severe was able to repeat words in a meaningless fashion (echolalia). The speech he uttered was more or less unintelligible (idioglossia); his comprehension of speech was better than in the former case. A child with an even milder degree of mental deficiency understood speech well but his speech was dyslalic. With training his speech became defective only in the articulation of single sounds (partial dyslalia).

The stages of retarded lingual development then range between mutism and normal speech and may be enumerated as follows: the articulation of primitive sounds, echolalia, idioglossia, universal dyslalia and partial dyslalia.

Manifestations of idioglossia.-The speech manifestations of retarded lingual development in any given stage are the same as those of normal lingual development in any comparable stage. The lingual phenomena of physiological idioglossia and pathological idioglossia therefore are the same. Inasmuch as idioglossia is merely a heightened form of dyslalia, a consideration of the manifestations of dyslalia ${ }^{11}$ will suffice. The following lingual phenomena will be observed: omission and reduction, substitutions, reduplication, assimilation, metathesis and analogy.

1. OMISSIQN AND REDUCTION are characterized by the omission of sounds and syllables from words, usually the difficult sounds and unaccented syllables. For instance, 'boom' is said for 'broom'; 'g'ome' for ' go home ';" ' pa-bu' for ' pocket-book.' 
2. Reduplication consists in the repetition of simple syllables to form words. For instance, ' moo-moo' is said for ' cow'; ' bow-bow' for ' dog.' These particular words imitate the sound of the thing indicated.

3. Assimilation. The child who says 'poom' for 'spoon' demonstrates assimilation combined with omission. Assimilation is the modification of one sound by another related in position or physiology. Thus ' $s$ ' is omitted from 'spoon,' and ' $\mathrm{n}$ ' is labialized to become ' $\mathrm{m}$ ' to conform to the labial sounds ' oo' and 'p.'

4. Substitution. The various sounds comprising the velar, labial and palatal groups are related in their mode of articulation. To one inexperienced in making these sounds substitutions are common. Those most frequently seen are: $d$ for $g$ (do for go); $t$ for $k$ (tat for cat); $d$ or $t$ for th (dis for this; tree for three); $f$ for th (free for three); $b$ for $v$ (bery for very); and $\mathrm{w}$ for $\mathrm{r}$ (wun for run). The following sentence demonstrates omissions of sounds and sound substitutions. 'Do 'ome an' ta' free tats wif oo' for ' go home and take three cats with you.'

5. Metathesis. Another manifestation of dyslalia is metathesis, which consists in the transposition of sounds or syllables in a word, or the transposition of words in a sentence. An example of transposition of sounds in a word is ' hopsital' for ' hospital.'

6. Analogy. Finally there is analogy which is the confusion of words due to a similarity in form, meaning or syntax (enemy for anemone; learn for teach; foots for feet), or the creation of new words or the application of new meanings to words by analagous association. Thus 'burneator' for ' radiator '; 'splinters' for ' moustache.'

\section{Analysis of cases.}

Physiologic idioglossia. Naomi S., aged $1 \frac{1}{2}$ years, comprehended speech well and used words with full comprehension of their meaning. These were more or less easily recognized if spoken singly. Otherwise her speech was idioglossic and quite unintelligible depending upon her loquacity. Especially at play one heard a flow of gibberish. During the following months, as her articulatory experience increased, a more normal speech began to evolve, and during this stage one was able to observe more readily the characteristic phenomena of dyslalia. Cmissions and reductions, sound substitutions and metatheses were common.

Idioglossia due to mental retardation. Dorothy E., aged 3. The mother of this child suffered from severe eclampsia during pregnancy and labour had to be induced. The infant was slow in sitting up and walking. At the age of three, speech was idioglossic although words might be articulated more or less clearly if her attention could be maintained. She repeated words and phrases that she heard in a meaningless fashion. Comprehension of speech was fairly good. Observation of her speech showed that she dropped the endings of words, assimilated, substituted and omitted sounds in order to simplify speech.

Idioglossia due to impaired hearing. Mary T., aged 7 , was a normal infant at birth. At the age of eight months she had a bilateral purulent otitis media which discharged for more than six months. She sat up at six months and walked at a year. During infancy her parents noticed that she paid no attention when spoken to, but it did not occur to them that her hearing was impaired. When she went to the hospital for diphtheria, they were told she did not hear. Her speech was mostly gibberish and she resorted to pantomime. She understood her mother, probably 
reading her lips. She has learned to say 'mother' and 'daddy' and several other words. 'Frances' was said as 'Fwances,' 'pretty' as 'pwetty,' 'silver' as 'sturber,' ' singac' as 'didac' or 'stigda.'

Kinesthetic idioglossia. An idioglossic child may be observed who is intelligent, whose comprehension of speech has developed normally, but whose articulation has remained faulty beyond the normal physiologic limits of three years. Such a child is not dysarthric, nor is the speech defect dependent on any peripheral organic defect of the organs of articulation. The fault lies probably in the motor speech area; this type of case may best be described by the term 'articulatory dyskinesthesia.' Doris Van S., aged 4, is a case in point. She was an intelligent child whose developmental history was normal as was the onset of speech. Comprehension of speech had always been good. Her speech was incomprehensible except to her mother. On analysis one observed the characteristic manifestations of dyslalia, notably sound substitutions. for instance, 'berry' was said for 'very '; 'wove' for 'love '; ' tat' for ' cat '; ' do' for ' go '; ' tit' for ' sit '; ete.

Idioglossia due to auditory imperception. Lastly, there is idioglossia due to congenital auditory imperception, which is comparable to the idioglossia just described in the motor sphere.

The diagnostic criteria in these cases are:-

1. Normal intelligence.

2. Normal hearing.

3. Failure to comprehend the meaning of words and often crude sounds.

4. Idioglossic or dyslalic speech.

5. The ability to acquire speech through motor and visual channels, such as lip reading.

A case of congenital auditory imperception reported by Morison ${ }^{9}$ may be cited. Wilfred C., aged 13, whose intelligence and hearing were normal, comprehended speech by lip reading. His own speech was idioglossic. 'Saturday night' was said as 'Tatur nigh,' 'September' as 'Temtember' ( $t$ substituted for $s$ in both these examples). 'Quarter past' said as 'tartar-tast' ( $t$ substituted for the $k$ sound, and $t$ used for $p$ in 'past' by progressive assimilation). 'Basket' was said as 'bassie' ( $k$ assimilated to $s$ and $t$ omitted). Note that although $t$ was substituted for $\mathrm{s}$ as in Temtember indicating an inability to articulate $s$, the ability to articulate this sound was shown in bassie for basket.

\section{Comment.}

Idioglossia then is a condition of speech reflecting the result of various influences to bring about phonetic change. Normally a faulty perception of new sound combinations and an articulatory inexperience combine to make the speech produced by the child in the first stages of speech acquisition idioglossic. As has been pointed out certain pathologic conditions may exist to interfere with the normal development of speech. The lingual phenomena observed are fundamentally the same. Cases of idioglossia or dyslalia, irrespective of the cause, reported by others may be studied and will be seen to display these lingual manifestations: omission and reduction, reduplication, assimilation, sound substitutions, metathesis, etc. Studying the speech defect in any given case will give no clue in itself to the underlying cause of the lingual defect. A history and examination of the patient along various lines will lead one to come to some conclusion concerning the nature 
of the retarding factor. This position has been admitted by Allen ${ }^{12}$, yet he would have us believe that true idioglossia is associated only with an inability to appreciate the meaning of word-sounds. In other words he says a defective condition of speech simulating idioglossia is observed under other circumstances than congenital auditory imperception, but these must be cases of spurious idioglossia. True idioglossia is characteristic of congenital auditory imperception alone but there is no means of distinguishing it from other forms of idioglossia by a study of the speech itself.

\section{Summary.}

Idioglossia is dyslalic speech so extreme in degree that it is incomprehensible to the ordinary listener. It is merely a stage in the development of speech whether this be normal or retarded. Its manifestations are the same as those of universal dyslalia: omission and reduction, reduplication, assimilation, metathesis, etc. Retarded lingual development of which idioglossia is a manifestation is due to impaired hearing, mental deficiency, articulatory dyskinesthesia and congenital auditory imperception. The idioglossia in these cases may be termed pathological idioglossia to distinguish it from the idioglossia of normal speech development or physiological idioglossia.

\section{REFERENCES.}

1. Hale White, W., \& Golding-Bird, C. H., Med.-Chir. Trans., Lond., 1891, LXXIV, 181.

2. McCready, E. B., Am. J. Psychiat., Baltimore, 1926, VI, 267.

3. Hadden, W. B., J. Ment. Sc., Lond, 1891, XXXVII, 96.

4. Thomas, C. J., Pub. Health, Lond., 1908, XXI, 90.

5. Wyllie, J., Disorders of Speech, Edin., 1894, 127.

6. Kerr, J., Brit. Med. J., Lond., 1900, i, 1231.

7. Guthrie, L., Functional Nervous Disorders in Children, Lond., 1907.

8. Worster-Drought, C., \& Allen, I. M., J. Neurol. \& Psychopath., Lond., 1930, $\mathrm{X}, 193$.

9. Morison, A. G., loc. cit., 28.

10. Barton Hall, S., \& Barton Hall, M., ibid., 1931, XI, 304.

11. Wolf, I. J., J. Med. Soc. New Jersey, Orange, 1934, XXXI, 156.

12. Allen, I. M., Brit. J. Child. Dis., Lond., 1932, XXIX, 98. 